\title{
Substituição do milho por polpa cítrica em rações com alta proporção de concentrado para cordeiros confinados
}

\author{
Replacement of corn by citrus pulp in high grain diets fed to feedlot lambs
}

\author{
Gustavo Henrique Rodrigues ${ }^{\mathrm{I}}$ Ivanete Susin $^{\mathrm{I}^{*}}$ Alexandre Vaz Pires ${ }^{\mathrm{I}}$ \\ Clayton Quirino Mendes ${ }^{I}$ Rafael Canonenco de Araujo ${ }^{I}$ \\ Irineu Umberto Packer ${ }^{I}$ Michele Ferreira Ribeiro ${ }^{I}$ \\ Luciana Vieira Gerage
}

\section{RESUMO}

A polpa cítrica desidratada (PC) é um subproduto altamente energético e com potencial para substituir o milho em rações de cordeiros desmamados precocemente. Dois experimentos foram realizados para avaliarem-se os efeitos da substituição do milho por PC no desempenho de cordeiros $e$ na digestibilidade das rações. No Experimento 1, 64 cordeiros Santa Inês, com peso vivo inicial de $18( \pm 0,6) \mathrm{kg}$ e $73( \pm 1)$ dias de idade, foram utilizados para avaliar o ganho de peso médio diário (GMD), o consumo de matéria seca (CMS) e a conversão alimentar (CA). No experimento 2, 12 cordeiros foram mantidos em gaiolas para ensaio de metabolismo para determinar a digestibilidade aparente das rações no trato digestório total e o metabolismo de nitrogênio. Nos dois experimentos, os cordeiros foram alimentados com uma ração contendo $90 \%$ de concentrado (milho moído elou PC, farelo de soja e minerais) e 10\% de feno de "coastcross" (Cynodon spp). A PC foi adicionada em 23,7; 46,1 e 68,4\% da MS, substituindo o milho em 33, 67 e 100\%, respectivamente. No Experimento 1, foi observado a maximização do GMD $\left(267 \mathrm{~g} \mathrm{dia}^{-1}\right)$ e do CMS $\left(1,01 \mathrm{~kg} \mathrm{dia}^{-1}\right)$, até o teor de substituição de $33 \%$ do milho pela PC. No Experimento 2, houve efeito linear $(P<0,05)$ decrescente na digestibilidade da MS $(P<0,03)$ e do $P B(P<0,10)$. Por outro lado, a digestibilidade da FDN aumentou linearmente com a adição da PC. Não houve diferença $(P>0,05)$ entre os tratamentos na digestiblidade aparente da matéria orgânica e no balanço de nitrogênio.

Palavras-chave: confinamento, pectina, Santa Inês, subprodutos

\section{ABSTRACT}

Dried citrus pulp (DCP) is a high energy byproduct and may be used to replace corn in early weaned lamb diets. Two trials were performed to evaluate the effects of replacing corn by dried citrus pulp on lamb performance and diet digestibility. In trial 1, 64 Santa Ines ram lambs (initial BW 18 $\pm 0.6 \mathrm{~kg}$ and $73 \pm 1$ days old) were used to evaluate average daily gain (ADG), dry matter intake (DMI) and feed conversion (FC). In trial 2, 12 ram lambs were placed in metabolism crates to evaluate $N$ metabolism and apparent digestibility of diets. In both trials, lambs were fed a $90 \%$ concentrate (ground corn and/or DCP, soybean meal and minerals) and $10 \%$ coastcross hay (Cynodon spp) diet. DCP was added at 23.7, 46.1 and $68 \%$ (diet DM) replacing corn by 33, 67 and $100 \%$, respectively. In Trial 1, the higher ADG $\left(267\right.$ day $\left.^{-1}\right)$ and DMI $\left(1.01 \mathrm{~kg}\right.$ day $\left.^{-1}\right)$ were observed when DCP replaced corn by $33 \%$ in the diet. In Trial 2, apparent digestibility of DM $(P<0.03)$ and $C P(P<0.10)$ showed a linear decrease. However, NDF digestibility increased linearly $(P<0.04)$ with the addition of $D C P$. OM digestibility and $N$ metabolism were similar among treatments.

Key words: byproducts, feedlot, pectin, Santa Inês.

\section{INTRODUÇÃO}

A crescente demanda por carne ovina registrada nos últimos anos impulsionou o aumento da produção de cordeiros para o abate, gerando a necessidade de melhoria nas técnicas de exploração. A adoção de algumas estratégias de produção intensiva faz-se necessária no agronegócio da ovinocultura, principalmente onde o custo da terra é elevado e a exigência do consumidor é cada dia mais acentuada.

Tradicionalmente, no programa nutricional de cordeiros confinados, o milho é um componente de destaque e de custo elevado. Por outro lado, os resíduos agroindustriais representam importantes recursos alimentares podendo reduzir o custo da alimentação.

\footnotetext{
'Departamento de Zootecnia, Escola Superior de Agricultura "Luiz de Queiroz” (ESALQ), Universidade de São Paulo (USP). Av.
} Pádua Dias, n 11, CP 9, 13418900, Piracicaba, São Paulo, Brasil. E-mail: ivasusin@esalq.usp.br. *Autor para correspondência. 
A polpa cítrica desidratada (PC) é um subproduto da indústria citrícola caracterizado por alto valor energético (13\% inferior ao do milho, segundo o NRC, 1996), com peculiaridades de fermentação que a coloco como produto intermediário entre volumoso e concentrado (FEGEROS et al., 1995). A PC é rica em açúcares (25\% na MS), fornecendo energia rapidamente disponível aos microrganismos ruminais, teor de amido reduzido, teor médio de fibra em detergente neutro (FDN) altamente digestível, e possuindo ainda na sua composição, principalmente pectina (NOCEK \& TAMMINGA, 1991).

A pectina é uma carboidrato estrutural complexo de alta e rápida degradação ruminal (VAN SOEST et al., 1991) e tem como unidade formadora o ácido galacturônico. As taxas de degradação ruminal da pectina, segundo SNIFFEN (1988), variam de 30 a 50\%/hora, enquanto o amido não processado a vapor é digerido em taxas que variam entre 10 a 20\%/hora. Contudo, a fermentação da PC no rúmen propicia produções maiores de acetato evitando queda brusca de $\mathrm{pH}$ ruminal, reduzindo assim os riscos de acidose por promover um ambiente ruminal mais favorável.

Aliada a essas características nutricionais, a época de produção da PC é favorável, tendo início em maio e término em janeiro, abrangendo justamente a entressafra de grãos como o milho e o período de escassez de forragem. Dessa forma, quando o milho atinge a cotação máxima e os pastos níveis mínimos de utilização, a PC representa uma forma de suplementação energética para essa época do ano. Portanto, a PC apresenta potencial para substituir parcial ou totalmente o milho em rações de alta proporção de concentrado destinadas a cordeiros confinados.

Há escassez de informações sobre o uso desse subproduto em substituição ao milho para cordeiros desmamados precocemente e alimentados com rações com alta proporção de concentrado. O objetivo deste estudo foi avaliar a substituição parcial ou total do milho moído por PC em rações contendo $90 \%$ de concentrado e $10 \%$ de feno de "coastcross", para cordeiros da raça Santa Inês em confinamento.

\section{MATERIAL E MÉTODOS}

Dois experimentos foram conduzidos no Sistema Intensivo de Produção de Ovinos e Caprinos (SIPOC) do Departamento de Zootecnia da Escola Superior de Agricultura “Luiz de Queiroz” (ESALQ), em Piracicaba, SP.

No experimento 1, foram utilizados 64 cordeiros da raça Santa Inês com peso médio inicial de $18( \pm 0,6) \mathrm{kg}$ e idade média de $73( \pm 1)$ dias. Os animais foram confinados por 56 dias em baias cobertas com piso de concreto, cocho e bebedouros, sendo distribuídos dois animais por baia e oito baias por tratamento. O delineamento experimental foi o de blocos completos casualizados com quatro tratamentos e oito repetições, sendo os blocos definidos pelo peso e pela idade inicial dos animais.

As rações foram formuladas para serem isonitrogenadas (16\% PB), constituídas de 10\% de volumoso (feno de "coastcross”) e $90 \%$ de concentrado na matéria seca (MS), incluindo a polpa cítrica na proporção de 23,7; 46,1 e 68,4\% da MS na ração, constituindo os tratamentos experimentais 0PC, 33PC, 67PC e 100PC, o que corresponde à substituição do milho nos teores de 0, 33, 67 e 100\%, respectivamente. A proporção dos ingredientes e a composição química das rações experimentais estão apresentadas na tabela 1 .

Em função da característica da ração (90\% MS), a alimentação foi fornecida três vezes/semana, ad libitum, e as sobras pesadas semanalmente para a determinação do consumo diário de MS por baia. Foi colhida uma amostra de cada partida das rações ofertadas e retirada uma alíquota semanal de $10 \%$ da sobra de cada baia (unidade experimental) e conservadas a $-10^{\circ} \mathrm{C}$. Posteriormente, as sobras foram descongeladas e compostas por baia. As amostras do alimento ofertado e das sobras foram processadas em moinho tipo Wiley, provido de peneira com crivos de $1 \mathrm{~mm}$ e analisadas para determinação da matéria seca, matéria mineral (MM) e proteína bruta (PB) de acordo com a AOAC (1990). A fibra em detergente neutro (FDN) foi determinada utilizando-se amilase e sulfito de sódio conforme VAN SOEST et al. (1991). A matéria orgânica foi calculada pela diferença entre a matéria seca e a matéria mineral.

No experimento 2, as rações experimentais foram as mesmas do experimento de desempenho. Os animais utilizados foram 12 ovinos da raça Santa Inês, com peso médio inicial de $36 \mathrm{~kg}$ e idade média inicial de cinco meses, em um delineamento experimental de blocos completos casualizados com quatro tratamentos e três repetições. Os animais foram alojados individualmente em gaiolas metálicas para ensaios de metabolismo com dimensões de 1,3 x 0,55m, providos de cochos e bebedouros. O período experimental teve a duração de 14 dias, sendo 10 dias de adaptação dos animais às instalações experimentais e quatro dias de colheita dos dados. Os animais foram pesados no início e no fim do período de colheita.

Os alimentos oferecidos e recusados e as fezes (coleta total) foram amostrados diariamente, às 16h, do oitavo ao décimo primeiro dia, e uma alíquota de $10 \%$ foi conservada a $-20^{\circ} \mathrm{C}$. Posteriormente, as 
Tabela 1 - Proporção dos ingredientes e composição química das rações experimentais (\% na MS).

\begin{tabular}{|c|c|c|c|c|}
\hline & \multicolumn{4}{|c|}{ Tratamentos $^{1}$} \\
\hline & OPC & 33PC & $67 \mathrm{PC}$ & $100 \mathrm{PC}$ \\
\hline \multicolumn{5}{|l|}{ Ingredientes } \\
\hline Feno de "coastcross" & 10,0 & 10,0 & 10,0 & 10,0 \\
\hline Milho moído & 70,4 & 47,2 & 23,4 & - \\
\hline Polpa cítrica & - & 23,7 & 46,1 & 68,4 \\
\hline Farelo soja & 16,0 & 17,0 & 17,9 & 18,2 \\
\hline Cloreto de amônio & 0,5 & 0,5 & 0,5 & 0,5 \\
\hline Calcário & 1,5 & - & - & - \\
\hline Monoaminofosfato & - & - & 0,5 & 1,3 \\
\hline Sal mineral $^{2}$ & 1,6 & 1,6 & 1,6 & 1,6 \\
\hline \multicolumn{5}{|l|}{ Composição bromatológica } \\
\hline Matéria seca & 88,8 & 89,4 & 89,9 & 91,4 \\
\hline Matéria mineral & 8,5 & 6,1 & 7,1 & 8,3 \\
\hline Matéria orgânica & 91,6 & 94,0 & 92,9 & 91,7 \\
\hline Proteína bruta & 17,5 & 17,1 & 17,9 & 17,3 \\
\hline Fibra em detergente neutro (FDN) & 19,5 & 20,4 & 24,2 & 24,6 \\
\hline
\end{tabular}

${ }^{1}$ Tratamentos: 0PC: sem substituição do milho pela polpa cítrica; 33PC: 33\% de substituição do milho pela polpa cítrica; 67PC: 67\% de substituição do milho pela polpa cítrica; 100PC: substituição total do milho pela polpa cítrica na MS da dieta.

${ }^{2}$ Composição: 7,5\% P, 19\% Ca; 1\% Mg; 7\% S; 14,3\% Na; 21,8\% Cl; 500ppm Fe; 300ppm Cu; 4600ppm Zn; 1100ppm Mn; 80ppm I; 405 ppm Co; 15ppm Se.

Todas as rações experimentais continham 25 ppm de monensina sódica para o controle da coccidiose.

amostras foram descongeladas, compostas por animal e secas em estufas de ventilação forçada $\left(55-60^{\circ} \mathrm{C}\right)$, por 72 horas, e procedidas as determinações de MS, MO, PB e FDN, como descrito anteriormente.

Os dados dos experimentos foram analisados pelo procedimento GLM do pacote estatístico SAS (1999). As médias das tabelas foram obtidas pelo comando LSMEANS. Para as variáveis que obtiveram respostas significativas $(\mathrm{P}<0,05)$, utilizou-se o teste para polinômios ortogonais (linear e quadrático).

\section{RESULTADOS E DISCUSSÃO}

Houve resposta quadrática $(\mathrm{P}<0,03)$ para o GMD, o CMS e a CA, ou seja, foi observada a maximização do CMS e do GMD até o teor de substituição de 33\% do milho pela PC (Tabela 2). Poucos trabalhos foram realizados especificamente com cordeiros recém desmamados para avaliar o GMD quando alimentados com esse subproduto. MARTINEZ \& FERNÁNDEZ (1980), MONTEIRO et al. (1998) e BUENO et al. (2004), trabalhando em dietas fornecendo 90, 80 e $68 \%$ de concentrado, utilizaram respectivamente a proporção máxima de 54,0, 25,3 e 40,5\% de PC na MS da dieta em substituição ao milho e não encontraram diferença no GMD e no CMS dos cordeiros. Entretanto, os valores de inclusão de PC foram menores quando comparados aos desse trabalho. No mesmo sentido, em um estudo utilizando PC desidratada ao sol, CAPARRA et al. (2007) não observaram efeito no desempenho quando incluíram a proporção máxima de $45 \%$.

No tratamento 33PC, onde houve a substituição de 33\% do milho por PC, os cordeiros apresentaram ganho de peso satisfatório $\left(267 \mathrm{~g} \mathrm{dia}^{-1}\right)$. No entanto, substituições maiores (67 e 100\%) afetaram consideravelmente o GMD, provavelmente devido à redução no consumo de MS, apresentando consumo abaixo da média recomendada pelo NRC (1985) para ovinos desta categoria (1,0 e 1,3kg MS animal $\left.{ }^{-1} \mathrm{dia}^{-1}\right)$. Além disso, pode ter ocorrido um efeito negativo sobre a absorção de Ca, P e Mg quando o milho foi substituído por grande quantidade de PC, causando alterações metabólicas (BUENO et al., 2002).

A substituição do milho pela PC em 33\% melhorou o consumo em relação à ração controle. Entretanto, a inclusão de PC nos teores de 46,1 e 68,4\% da MS (67 e 100\% de substituição) na ração influenciou negativamente no CMS. Os valores encontrados no presente estudo para CMS variaram entre 0,843 e $1,007 \mathrm{~kg}_{\text {animal }}{ }^{-1} \mathrm{dia}^{-1}$, podendo justificar os resultados de desempenho dos animais. Porém, não foi verificado efeito da inclusão de PC no CMS quando expresso em função do peso metabólico do animal, concordando com os dados do experimento de digestibilidade. 
Tabela 2 - Ganho médio diário (GMD), consumo de matéria seca (CMS) e conversão alimentar (CA) dos cordeiros no período experimental.

\begin{tabular}{|c|c|c|c|c|c|c|}
\hline \multicolumn{7}{|l|}{ Tratamentos } \\
\hline Item & & 33PC & $67 \mathrm{PC}$ & 100PC & $\mathrm{EPM}^{2}$ & $\mathrm{P}^{3}$ \\
\hline Número de animais & 16 & 16 & 16 & 16 & & \\
\hline Idade inicial, dias & 71 & 74 & 73 & 72 & 1,4 & 0,35 \\
\hline Idade final, dias & 127 & 130 & 129 & 128 & 1,2 & 0,36 \\
\hline Peso inicial, kg & 18,0 & 17,9 & 18,0 & 18,0 & 0,6 & 0,48 \\
\hline Peso final, $\mathrm{kg}^{4}$ & 32,1 & 32,9 & 29,4 & 27,3 & 0,9 & $<0,01$ \\
\hline GMD, $\mathrm{g}^{5}$ & 253,3 & 267,0 & 203,5 & 166,9 & 11,0 & $<0,01$ \\
\hline \multicolumn{7}{|l|}{ CMS } \\
\hline$g / d^{6}$ & 943,2 & 1007,5 & 859,7 & 843,3 & 30,5 & 0,01 \\
\hline $\mathrm{g} / \mathrm{kg}\left(\mathrm{PV}^{0.75}\right)$ & 84,8 & 88,8 & 80,3 & 80,9 & 2,2 & 0,08 \\
\hline$\% \mathrm{PV}$ & 3,8 & 4,0 & 3,6 & 3,7 & 0,1 & 0,17 \\
\hline $\mathrm{CA}, \mathrm{kg} \mathrm{MS} / \mathrm{kg}$ ganho $^{7}$ & 3,7 & 3,8 & 4,3 & 5,3 & 0,2 & $<0,01$ \\
\hline
\end{tabular}

${ }^{1}$ Tratamentos: 0PC: sem inclusão de polpa cítrica na dieta; 33PC: 33\% de substituição do milho pela polpa cítrica; 67PC: 67\% de substituição do milho pela polpa cítrica; 100PC: substituição total do milho pela polpa cítrica na dieta.

${ }^{2}$ Erro padrão da média.

${ }^{3}$ Efeito de tratamento.

${ }^{4} \mathrm{Y}=32,807-0,0055 \mathrm{X}-0,0005 \mathrm{X}^{2}\left(\mathrm{P}<0,03 ; \mathrm{R}^{2}=0,49\right)$.

${ }^{5} \mathrm{Y}=262,1+0,1 \mathrm{X}-0,0109 \mathrm{X}^{2}\left(\mathrm{P}<0,01 ; \mathrm{R}^{2}=0,62\right)$.

${ }^{6} \mathrm{Y}=967,4+0,2 \mathrm{X}-0,0163 \mathrm{X}^{2}\left(\mathrm{P}<0,01 ; \mathrm{R}^{2}=0,26\right)$.

${ }^{7} \mathrm{Y}=3,7152-0,0066 \mathrm{X}+0,0002 \mathrm{X}^{2}\left(\mathrm{P}<0,02 ; \mathrm{R}^{2}=0,55\right)$.

A conversão alimentar do presente estudo apresentou efeito quadrático $(\mathrm{P}<0,02)$. A substituição total do milho pela PC (100PC) piorou a conversão alimentar (5,31 $\mathrm{kg} \mathrm{CMS} \mathrm{kg}^{-1}$ ganho). Esses dados diferem dos resultados obtidos por BUENO et al. (2004), que não observaram alterações na conversão alimentar de cordeiros alimentados com rações contendo PC. Entretanto, o valor máximo de inclusão no presente trabalho (68\% da MS) é superior ao teor máximo (40,5\% da MS) utilizado por BUENO et al. (2004). Por outro lado, MARTINEZ \& FERNÁNDEZ (1980) verificaram redução linear na conversão alimentar de cordeiros alimentados com rações contendo PC, sendo $60 \%$ da MS o teor máximo de inclusão de PC, concordando com CAPARRA et al. (2007), que encontraram maior eficiência alimentar para rações contendo 45\% de PC desidratada ao sol.

No presente estudo, foi observado efeito linear decrescente na digestibilidade da MS $(\mathrm{P}<0,04) \mathrm{e}$ de $\mathrm{PB}(\mathrm{P}<0,10)$ e crescente para a FDN $(\mathrm{P}<0,04)$. Adicionalmente, não houve diferença $(\mathrm{P}>0,05)$ entre os tratamentos na digestiblidade aparente da matéria orgânica (Tabela 3).

A diminuição linear $(\mathrm{P}<0,05)$ do consumo de MS foi acompanhada pela queda linear $(\mathrm{P}<0,05) \mathrm{da}$ digestibilidade de MS. Por outro lado, HENRIQUE et al. (2003), utilizando ovinos mestiços com 9 meses de idade e $35 \mathrm{~kg}$ de peso vivo, alimentados com teores crescentes de PC (0, 25, 40 e 55\% da MS) em rações contendo $20 \%$ de silagem de milho, verificaram aumento linear nos coeficientes de digestibilidade da PB e nenhuma alteração na digestibilidade da MS. Os autores sugerem que esses resultados tenham sido obtidos pelo efeito associativo entre os ingredientes nas rações com a inclusão de PC, melhorando o ambiente ruminal e o aproveitamento desses nutrientes. Da mesma forma, BHATTACHARYA \& HARB (1973) não encontraram diferença na digestibilidade aparente da MS de rações com $10 \%$ de volumoso e teores crescentes de PC.

Segundo WAINMAN \& DEWEY (1988), a digestibilidade da proteína da PC é considerada baixa (40 a 65\%), caracterizando produtos que sofreram secagem, o que explica o decréscimo linear $(\mathrm{P}<0,10)$ observado na digestibilidade da proteína bruta com o aumento da inclusão de PC, variando de $68 \%$ para o tratamento 0PC a $62 \%$ para o tratamento 100PC. Esses resultados concordam com os obtidos por BENGHEDALIA et al. (1989), que verificaram diminuição da digestibilidade aparente do nitrogênio em rações contendo PC, em comparação a rações contendo cevada. Por outro lado, esse mesmos resultados contrastam com BUENO et al. (2002), que não verificaram efeito do subproduto para essa variável.

A digestilbilidade aparente da FDN apresentou efeito linear $(\mathrm{P}<0,04)$ crescente com $\mathrm{o}$ acréscimo de PC nas rações, variando de $48,4 \%$ no tratamento controle a $61,2 \%$ no tratamento em que o milho foi substituído totalmente pela PC.

Esses resultados concordam com os apresentados por BUENO et al. (2002), que, ao fornecerem quantidades crescentes de PC (máxima 
Tabela 3 - Consumo e digestibilidade aparente no trato digestório (DATD) da matéria seca (MS), matéria orgânica (MO), fibra em detergente neutro (FDN), proteína bruta (PB) e balanço de nitrogênio das rações experimentais.

\begin{tabular}{|c|c|c|c|c|c|c|}
\hline \multirow[b]{2}{*}{ Item } & \multicolumn{4}{|c|}{ Tratamentos $^{1}$} & \multirow{2}{*}{ EPM } & \multirow{2}{*}{ Efeito linear } \\
\hline & OPC & 33PC & 67PC & $100 \mathrm{PC}$ & & \\
\hline & \multicolumn{6}{|c|}{ Matéria seca } \\
\hline Consumo, $g / \mathrm{d}^{3}$ & 1200,1 & 1047,6 & 1039,4 & 902,4 & 70,1 & 0,02 \\
\hline Consumo, $\mathrm{g} / \mathrm{kg}\left(\mathrm{PV}^{0,75}\right)$ & 81,1 & 70,2 & 70,7 & 65,4 & 4,5 & 0,25 \\
\hline \multirow[t]{2}{*}{ DATD, $\%^{4}$} & 77,1 & 77,5 & 76,0 & 71,9 & 1,4 & 0,03 \\
\hline & \multicolumn{6}{|c|}{ Matéria orgânica } \\
\hline Consumo, g/d ${ }^{5}$ & 1099,1 & 976,0 & 956,7 & 838,4 & 60,2 & 0,03 \\
\hline Consumo, $\mathrm{g} / \mathrm{kg}\left(\mathrm{PV}^{0,75}\right)$ & 73,7 & 65,9 & 65,7 & 59,6 & 4,3 & 0,39 \\
\hline \multirow[t]{2}{*}{ DATD, \% } & 77,8 & 78,3 & 77,4 & 74,3 & 1,4 & 0,12 \\
\hline & \multicolumn{6}{|c|}{ Fibra em detergente neutro } \\
\hline Consumo, g/d & 250,3 & 230,1 & 280,9 & 260,5 & 23,5 & 0,44 \\
\hline Consumo, $\mathrm{g} / \mathrm{kg}\left(\mathrm{PV}^{0,75}\right)$ & 17,1 & 15,4 & 19,3 & 18,8 & 1,4 & 0,65 \\
\hline \multirow[t]{2}{*}{ DATD, $\%^{6}$} & 48,1 & 50,8 & 59,4 & 61,2 & 4,1 & 0,04 \\
\hline & \multicolumn{6}{|c|}{ Proteína bruta } \\
\hline Consumo, g/d ${ }^{7}$ & 210,2 & 180,9 & 190,7 & 170,3 & 11,1 & 0,05 \\
\hline Consumo, $\mathrm{g} / \mathrm{kg}\left(\mathrm{PV}^{0,75}\right)$ & 14,5 & 12,1 & 13,3 & 12,3 & 0,7 & 0,65 \\
\hline DATD, $\%^{8}$ & 67,7 & 65,5 & 66,7 & 62,1 & 1,8 & \\
\hline $\mathrm{N}$ fezes, g/d & 9,7 & 8,6 & 8,9 & 9,0 & 0,5 & 0,54 \\
\hline $\mathrm{N}$ urina, g/d & 6,8 & 7,0 & 5,7 & 6,4 & 1,3 & 0,88 \\
\hline \multicolumn{7}{|l|}{ Balanço de N } \\
\hline$g / d$ & 17,9 & 12,9 & 16,3 & 11,8 & 1,9 & 0,20 \\
\hline$\% \mathrm{~N}$ consumido & 52,0 & 45,5 & 52,3 & 43,3 & 5,0 & 0,52 \\
\hline \% N absorvido & 76,7 & 69,2 & 78,4 & 69,8 & 7,1 & 0,73 \\
\hline
\end{tabular}

${ }^{1}$ Tratamentos: 0PC: sem inclusão de polpa cítrica na ração; 33PC: 33\% de substituição do milho pela polpa cítrica; 67PC: 67\% de substituição do milho pela polpa cítrica; 100PC: substituição total do milho pela polpa cítrica na ração.

${ }^{2}$ Erro Padrão da Média.

${ }^{3} \mathrm{Y}=1176,1-2,614 \mathrm{X}\left(\mathrm{R}^{2}=0,43\right)$.

${ }^{4} \mathrm{Y}=78,184-0,0513 \mathrm{X}\left(\mathrm{R}^{2}=0,43\right)$.

${ }^{5} \mathrm{Y}=1083,2-2,4467 \mathrm{X}\left(\mathrm{R}^{2}=0,42\right)$.

${ }^{6} \mathrm{Y}=47,66+0,1442 \mathrm{X}\left(\mathrm{R}^{2}=0,51\right)$

${ }^{7} \mathrm{Y}=206,7-0,3556 \mathrm{X}\left(\mathrm{R}^{2}=0,28\right)$.

${ }^{8} \mathrm{Y}=67,823-0,0465 \mathrm{X}\left(\mathrm{R}^{2}=0,28\right)$.

inclusão de 66,5\%) para cabritos da raça Saanen, verificaram aumento linear na digestiblidade da FDN, e por BHATTACHARYA \& HARB (1973), que observaram aumento proporcional na digestibilidade da porção fibrosa até o teor de $60 \%$, na ração de ovinos, à medida que o milho foi substituído pela PC. FEGEROS et al. (1995), trabalhando com ovelhas recebendo rações constituídas de feno e $30 \%$ de PC, encontraram valores de 87,$2 ; 78,6 ; 52,7 ; 82,0 ; 93,2$; e $83,1 \%$ para a digestibilidade aparente das frações MO, MS, PB, EE, FB e ENN, respectivamente, verificando aumento na digestibilidade da FB em rações contendo até 30\% de PC.

BEN-GHEDALIA et al. (1989) observaram que a digestibilidade dos constituintes da parede celular (arbinose, galactose, manose e xilose) é $16 \%$ superior nas rações contendo PC em relação àquelas contendo cevada e constituídas de $80 \%$ de concentrado, o que pode explicar a melhora na digestibilidade da fibra. VAN SOEST (1982) sugere melhor padrão de fermentação no rúmen, propiciando condições para a atuação das bactérias celulolíticas em rações contendo PC, facilitando a ação desses microorganismos à digestão da parede celular.

Não foi verificada diferença $(\mathrm{P}>0,05)$ na quantidade de $\mathrm{N}$ nas fezes e na urina quando a $\mathrm{PC}$ foi adicionada na ração, não alterando o metabolismo de $\mathrm{N}$, o que concorda com dados apresentados por ESTEVES et al. (1987) e BUENO et al. (2002), que substituíram milho por PC em rações para bovinos e caprinos, respectivamente. Por outro lado, os resultados observados por HENRIQUE et al. (2003) mostraram melhor aproveitamento do $\mathrm{N}$ em ovinos recebendo PC, justificado pelo aumento da ingestão de MS com a adição de PC. Alguns trabalhos ainda demonstraram diminuição significativa do nitrogênio 
retido como o aumento da participação da PC na ração (BHATTACHARYA \& HARB, 1973; PASCUAL \& CARMONA, 1980).

\section{CONCLUSÕES}

A substituição de um terço do milho por polpa cítrica melhora o consumo de matéria seca e o desempenho de cordeiros alimentados com alta proporção de concentrado. Entretanto, a adição de polpa cítrica diminui a digestibilidade aparente da MS e da PB, com aumento da digestibilidade da FDN, não alterando o metabolismo de nitrogênio.

\section{AGRADECIMENTOS}

À Coordenação de Aperfeiçoamento de Pessoal de Nível Superior (CAPES), pela bolsa de mestrado concedida ao primeiro autor, e à Fundação de Amparo à Pesquisa do Estado de São Paulo (FAPESP), pelo financiamento parcial do projeto.

\section{REFERÊNCIAS}

ASSOCIATION OF OFFICIAL ANALYTICAL CHEMISTS. Official methods of analysis. 15ed. Arlington, 1990. V.1, 1117p.

BEN-GHENDALIA et al. The effects of starch and pectin rich diets on quantitative aspects of digestion in sheep. Animal Feed Science and Technology, v.24, p.289-298, 1989.

BHATTACHARYA, A.N.; HARB, M. Dried citrus pulp as grain replacement for Awassi lambs. Journal of Animal Science, v.36, n.6, p.1175-1180, 1973.

BUENO, M.S. et al. Polpa cítrica desidratada na dieta de borregos Suffolk e Santa Inês, em confinamento. Boletim da Indústria Animal, v.61, n.2, p.115-119, 2004.

BUENO, M.S. et al. Effect of replacing corn with dehydrated citrus pulp in diets of growing kids. Small Ruminant Research, v.46, p.179-185, 2002.

CAPARRA, P. et al. Solar-dried citrus pulp as an alternative energy source in lamb diets: effects on growth an carcass and meat quality. Small Ruminant Research, v.40, n.3, p.303311, 2007.

ESTEVES, S.N. et al. Substituição da espiga de milho desintegrada com palha e sabugo pela polpa de citros peletizada na engorda de bovinos Canchim. Revista da Sociedade Brasileira de Zootecnia, v.16, n.6, p.507-516, 1987.

FEGEROS, K. et al. Nutritive value of dried citrus pulp and its effect on milk yield and milk composition of lactating ewes. Journal of Dairy Science, v.78, n.5, p.1116-1121, 1995.

HENRIQUE, W. et al. Digestibilidade e balanço de nitrogênio em ovinos alimentados à base de dietas com elevado teor de concentrado e níveis crescentes de polpa cítrica peletizada. Revista Brasileira de Zootecnia, v.32, n.6, p.2007-2015, 2003 (supl.2).

MARTINEZ, P.J.; FERNÁNDEZ, C.J. Citrus pulp in diets for fattening lambs. Animal Feed Science and Technology, v.5, n.1, p.11-22, 1980.

MONTEIRO, A.L.G. et al. Desempenho e características quantitativas das carcaças de cordeiros alimentados com polpa cítrica em substituição ao milho. Unimar Ciências, v.7, n.1, p 65-70, 1998.

NATIONAL RESEARCH COUNCIL. Nutrient requirements of sheep. 6.ed. Washington: National Academic, 1985. 99p.

NATIONAL RESEARCH COUNCIL. Nutrient requirements of beef cattle. 7.ed. Washington: National Academic, 1996. 242p.

NOCEK, J.E.; TAMMINGA, S. Site of digestion of starch in the gastrointestinal tract of dairy cows and its effect on milk yield and composition. Journal of Dairy Science, v.74, p.3598-3629, 1991.

PASCUAL, J.M.; CARMONA, J.F. Composition of citrus pulp. Animal Feed Science and Technology, v.5, p.1-10, 1980.

SNIFFEN, C.J. Balancing rations for carbohydrates for dairy cattle. In: FEED DEALER SEMINARS, 1998, New York. Proceedings... Cornell: Cornell Cooperative Extension, 1988. N.112, p.9-19.

STATISTICAL ANALYSIS SYSTEM - SAS. User's guide: statistic. 6.ed. Cary, 1999. 956p.

VAN SOEST, P.J. Nutritional ecology of the ruminant. Corvallis: O\& Books, 1982. 373p.

VAN SOEST, P.J. et al. Methods for dietary fiber, neutral detergent fiber, and non starch polysaccharides in relation to animal nutrition. Journal of Dairy Science, v.74, p.35833597, 1991.

WAINMAN, F.W.; DEWEY, J.S. Feedingstuffs evaluation unit - fifth report. Bucksburn, Scotland, UK: Rowett Research Institute, 1988. 123p. 